\title{
Médiévales
}

Langues, Textes, Histoire

68 | printemps 2015

Langues d'Angleterre

\section{Paolo PIVA (dir.), Art médiéval. Les voies de l'espace}

\section{liturgique}

Paris, Picard, 2010, 288 p.

\section{Pierre-Olivier Dittmar}

\section{(2) OpenEdition}

Journals

\section{Édition électronique}

URL : https://journals.openedition.org/medievales/7502

DOI : $10.4000 /$ medievales. 7502

ISSN : 1777-5892

Éditeur

Presses universitaires de Vincennes

\section{Édition imprimée}

Date de publication : 15 juin 2015

Pagination : 181-185

ISBN : 978-2-84292-430-0

ISSN : 0751-2708

Référence électronique

Pierre-Olivier Dittmar, « Paolo piva (dir.), Art médiéval. Les voies de l'espace liturgique », Médiévales [En ligne], 68 I printemps 2015, mis en ligne le 09 juillet 2015, consulté le 24 avril 2022. URL : http:// journals.openedition.org/medievales/7502; DOI : https://doi.org/10.4000/medievales.7502

Ce document a été généré automatiquement le 24 avril 2022

Tous droits réservés 


\title{
Paolo PIVA (dir.), Art médiéval. Les voies de l'espace liturgique
}

Paris, Picard, 2010, 288 p.

\author{
Pierre-Olivier Dittmar
}

\section{RÉFÉRENCE}

Paolo PIVA (dir.), Art médiéval. Les voies de l'espace liturgique, Paris, Picard, 2010, 288 p.

1 L'apparence de cet ouvrage est trompeuse. Son titre (Art médiéval), le nombre et la qualité des reproductions, ainsi que son prix (91 euros), le présentent comme un «beau livre » davantage destiné à orner les salons qu'à être lu. Ce serait particulièrement dommage dans la mesure où cet important travail dirigé par P. Piva est amené à faire date en raison de ses qualités scientifiques. Il propose en effet, et pour la première fois, une synthèse des recherches menées par un certains nombre de chercheurs européens sur les liens associant l'image, l'espace et le rituel.

2 L'ouvrage peut être sommairement divisé en deux parties. Une première série d'articles se concentre sur l'architecture et le mobilier liturgique du haut Moyen Âge à l'époque romane. Ouvrant cette séquence chronologique, l'important essai de Sible de Blaauw se penche sur la passionnante question de l'orientation des édifices chrétiens. Le sujet est ici abordé de façon virtuose et audacieuse, traitant successivement des dimensions architecturales, liturgiques et symboliques. Contrairement à l'impression chaotique que donnent les orientations des premières églises romaines, l'auteure montre comment se met en place un dispositif spécifiquement chrétien jouant sur trois facteurs : l'alignement de l'axe principal de l'édifice (est-ouest), le primat donné à la logique interne de l'édifice sur les considérations urbanistiques et extérieures (notamment en ce qui concerne la lumière), et la direction selon laquelle l'office est célébré. Dès lors, si l'axe est-ouest est autant que possible respecté - notamment en raison d'un symbolisme solaire bien ancré - et si l'orientation du prêtre vers l'est est indispensable, les autres facteurs peuvent largement être modifiés en fonction des 
contraintes et des traditions locales, ce qui aboutit à des solutions où l'officiant peut soit être tourné vers les fidèles, soit leur tourner le dos. Ce dispositif, qui connait de nombreuses et passionnantes variations, ne résiste pas à la multiplication des autels privés qui rendent l'orientation du culte impossible. Aux $\mathrm{XV}^{\mathrm{e}}-\mathrm{XVI}^{\mathrm{e}}$ siècles, c'est de plus en plus le contexte urbanistique qui impose son orientation à l'édifice, et la position de l'autel qui détermine abstraitement l'orientation liturgique: signes manifestes d'une remise en cause du symbolisme cosmologique si important au Moyen Âge.

Werner Jacobsen propose, quant à lui, une très utile synthèse des différentes formes architecturales (et souvent des dispositifs liturgiques) du haut Moyen Âge à partir d'un ensemble de 2000 édifices répartis dans toute l'Europe et dont on parvient à connaître les plans d'origine. On peut saluer le tour de force tant cette période se distingue par son inventivité architecturale, rétive à toute réduction. Certaines évolutions peuvent malgré tout être dégagées : la construction aux $\mathrm{VI}^{\mathrm{e}}$-VII ${ }^{\mathrm{e}}$ siècles, à Rome, des églises SaintLaurent et Sainte-Agnès implique de considérables travaux de terrassement pour construire les églises au-dessus des tombeaux des saints. Elle témoigne en cela d'un rapport nouveau au lieu, contrastant avec les pratiques antérieures où l'on déplaçait plus volontiers les restes des saints. Dans ce type de dispositif, l'autel est désormais à la verticale de la sépulture, ce qui entraîne un nouvel aménagement du chœur et, par voie de conséquence, l'invention de la crypte, qui est une singularité particulièrement marquante des dispositifs liturgiques médiévaux. Mais même ces grandes tendances ne peuvent acquérir un caractère de généralité. De fait, l'originalité des partis pris architecturaux est chaque fois issue de la combinaison d'une série de contraintes dont chacune peut devenir prépondérante, qu'il s'agisse des traditions locales (franques, germaniques...) ou de choix esthétiques qui sont aussi de claires prises de positions politiques (pro-romains ou anti-romains), sans oublier de véritables inventions d'architecte répondant aux exigences pratiques du rite spécifique à chaque édifice. Une telle variété peut être rapprochée de la fragmentation politique de l'ensemble de l'Occident qui favorise les affirmations et les innovations locales. À ce titre, il aurait été instructif de comparer cette diversité des plans avec la remarquable stabilité de l'architecture byzantine ordinaire dans les siècles entourant l'an mil.

4 À partir d'un corpus proche, Paolo Piva associe de nombreux plans, la littérature liturgique et les résultats de fouilles (comme dans le cas passionnant de l'église de Charroux, à la fin du xI ${ }^{\mathrm{e}}$ siècle), et questionne plus directement les usages sociaux des églises, en se concentrant sur deux innovations majeures de l'architecture religieuse médiévale: le déambulatoire et la crypte. En critiquant de façon particulièrement convaincante l'idée - issue du $\mathrm{XIX}^{\mathrm{e}}$ siècle - d'un ensemble d'églises de pèlerinage qui possèderaient des traits architecturaux communs, l'auteur montre que l'on ne peut attribuer aux tribunes et aux déambulatoires la (seule) fonction d'accueillir les pèlerins. En effet, il apparaît qu'indépendamment des pèlerinages qu'elle peut accueillir, c'est le statut de l'église (canoniale, monastique, etc.) qui s'avère déterminant pour appréhender les fonctions de ses différentes parties. De fait, s'il n'y a pas d'« église de pèlerinage ", il existe bien des "parcours de pèlerinage » qui viennent s'insérer dans les dispositifs spécifiques à chaque édifice. Ici aussi la prégnance des modèles romains joue un rôle de premier plan, les architectures à crypte s'inscrivant dans la filiation de Saint-Pierre de Rome, alors que des parcours plus longitudinaux font davantage référence à Saint-Paul. Au fil des exemples, le déambulatoire s'impose comme un espace de dégagement permettant avant tout de multiplier les autels et les messes 
privées, mais aussi de relier des espaces sans passer par un sanctuaire où l'on officie en permanence. Quand bien même le déambulatoire peut être utilisé lors de processions, sa présence dépend avant tout de la volonté plus ou moins grande de séparer la circulation des clercs et celle des laïcs, une séparation matérialisée par la présence de barrières liturgiques dont l'emplacement est décisif pour qui veut saisir l'usage des édifices religieux chrétiens. En dernier lieu, il convient de ne pas réduire ces architectures à un pur fonctionnalisme : si le critère esthétique ne peut être considéré comme étant le seul déterminant, il est manifeste qu'il intervient dans les choix architecturaux, et que la présence d'une abside à chapelles rayonnantes doit aussi se penser dans un contexte d'émulation où cette forme permet d'affirmer avec vigueur les prétentions des commanditaires.

5 La seconde partie de l'ouvrage couvre les périodes romane et gothique, et correspond à un contexte où les formes architecturales se sont (un peu) stabilisées; c'est alors bien davantage les images peintes ou sculptées qui deviennent l'élément de variabilité, l'espace d'expression des commanditaires et le marqueur des partis pris locaux. Il est d'ailleurs manifeste que l'approche de la liturgie change de statut dans cette partie de l'ouvrage. Il s'agit ici de penser non plus directement les lieux où elle prend place, mais la nature des liens entre les images présentes dans ces édifices et les rituels qui s'y déroulent. Marcello Angheben propose ainsi un parcours critique des principales hypothèses en la matière; en attendant la multiplication d'analyses sérielles permettant l'analyse «syntaxique» que l'auteur appelle plusieurs fois de ses vœux, l'état de l'art ne permet pas encore de dégager des principes généraux. Ainsi, si des différences thématiques ou qualitatives reflètent parfois l'investissement relatif des différents espaces (notamment en donnant une importance plus grande au massif oriental), ces distinctions peuvent aussi être le fruit de l'histoire d'un chantier. Certes, il est possible de constater que les scènes animales ou diaboliques, violentes, se font plus rares à l'approche du sanctuaire, mais la prudence de l'auteur l'interdit d'aller audelà de ce constat a minima du lien entre dispositif liturgique et répartition des sculptures dans l'édifice. En tout état de cause, il est particulièrement rare que les sculptures représentent (ou même évoquent) directement des rituels à l'époque romane; tout au plus certains chapiteaux intègrent-ils des éléments iconographiques se référant à des passages bibliques et évangéliques ayant pu être "activés» lors d'actions liturgiques, mais ils ne peuvent en aucun cas être réduits à cette fonction. C'est avant tout la peinture, avec la représentation de théophanies dans les conques absidiales des églises romanes, qui offre le meilleur exemple d'usage liturgique du décor, puisque la figuration du Christ en gloire permet de manifester sa présence au début du canon de la messe (bien plus que son retour à la fin des temps comme on l'interprète généralement). C'est d'ailleurs à l'aune de ces images peintes que sont interprétés les programmes sculptés de nombreux portails romans. Plutôt que de chercher un lien avec des rituels qui se seraient déroulés devant la façade de l'église, l'auteur suggère de les comprendre comme l'annonce, à l'extérieur, de la présence divine figurée à l'intérieur. Ce faisant, c'est une approche «feuilletée » du décor qui est proposée, composée d'une série de seuils (façade, barrière liturgique, sanctuaire) dont les thèmes se répondent.

6 Le texte de Jérôme Baschet prolonge cette réflexion en l'infléchissant sensiblement. L'auteur offre au lecteur plusieurs prises de distance vis-à-vis de l'objet-église qui donnent à cette contribution une remarquable qualité de synthèse. Distance thématique tout d'abord: si les images et l'architecture ne peuvent être dissociées de la liturgie 
qu'elles accompagnent, cette dernière ne doit pas non plus être considérée en dehors de la société qui la produit et la pratique. C'est pourquoi ces images gagnent à être pensées à partir des acquis de l'histoire sociale, qui a bien décrit le processus de monumentalisation des édifices de culte et l'importance toujours grandissante qu'occupe le lieu dans l'exercice de la liturgie. Distance chronologique ensuite : c'est en observant la forme du bâtiment ecclésial dans la longue durée que les évolutions macroscopiques apparaissent clairement. À mesure que la bipolarité carolingienne s'estompe, l'importance de l'axe longitudinal, qui prend l'autel majeur comme point d'aboutissement, s'affirme comme une caractéristique occidentale de l'architecture chrétienne. Cette dimension axiale joue un rôle central dans la compréhension de ces édifices; elle s'impose comme la matérialisation d'un Iter, c'est-à-dire d'un chemin de vie et de conversion, depuis le monde profane jusqu'au lieu du sacrifice.

7 Trois critères apparaissent donc décisifs dans la compréhension de l'édifice ecclésial : d'une part, l'unité sacrale du lieu (s'affirmant, dans son ensemble, comme étant la maison de Dieu); d'autre part, les divisions internes précédemment évoquées par $\mathrm{M}$. Angheben (où la répartition des espaces liturgiques ne correspond pratiquement jamais aux divisions lisibles dans l'architecture); enfin, une dynamique axiale traversant tout l'édifice, ce qui conduit à le penser non pas comme une série de zones statiques, mais comme un lieu travaillant expressément une déambulation spiritualisante. Ce dernier aspect est à la fois le plus orignal et le plus important : le prendre en compte constitue le meilleur antidote pour se garder de toute lecture dualiste de l'église, qui opposerait une nef assimilée au monde profane à un sanctuaire totalement positif. Si ce dernier lieu constitue bien un pôle attractif, doté d'une grande sacralité, le lieu de rencontre entre la liturgie céleste (représentée dans l'abside) et la liturgie terrestre, il est indispensable de considérer que c'est l'église tout entière qui constitue un Iter et s'impose comme un lieu liminaire. Ce dernier aspect est remarquablement démontré dans l'analyse du programme peint de l'église de SaintSavin, qui donne l'occasion à l'auteur de proposer une sorte d'iconographie totale du lieu sacré, les aspects ornementaux et les motifs végétaux, généralement oubliés par les études iconographiques, jouant un rôle de premier plan et permettant de comprendre la logique du dispositif liturgique de cet édifice. La dernière contribution du volume contient un essai passionnant, mais problématique par certains aspects, consacré à la question liturgique dans le corpus figuratif des cathédrales gothiques. Si le lecteur est parfois un peu décontenancé, c'est surtout parce qu'à la différence des deux interventions précédentes, la liturgie est prise ici dans une acception vague et large, ce qui pose un certain problème de corpus. Il est en effet indéniable qu'à un certain niveau de généralité, toute image chrétienne peut être rattachée à des préoccupations liturgiques: toute image du Christ peut ainsi - à l'extrême limite - être considérée comme renvoyant à l'eucharistie. À ce titre, la présence des vies de saints sur les vitraux des cathédrales fait certes écho à la présence de leurs reliques dans l'édifice, mais cela nous renseigne bien peu sur leur usage rituel. Dans un tout autre registre, l'interprétation psychologisante de l'homme médiéval, qui devait «naturellement rire et sourire" de l'image grotesque du diable pour mieux surmonter la crainte que suscitait le temps de la passion, est loin d'être convaincante (p. 259). Mais il serait mesquin de s'en tenir à ces quelques réserves tant le dossier présenté est par ailleurs riche en érudition, précis et stimulant dans les analyses. C'est notamment le cas de l'analyse des portails de la cathédrale d'Amiens dédiés à saint Firmin et saint Honoré, qui, par le recoupement de l'image et des sources liturgiques, notamment l'ordinaire de 
1291, permet d'approcher cette forme d'art total que constituent les grandes fêtes liturgiques. À Amiens, ce n'est pas la vie de saint Firmin, mais bien le fantastique dispositif liturgique que constitue la translation des reliques qui est représenté en façade de la cathédrale, fournissant un rare exemple de mise en scène en image, dans l'espace public, d'une série d'actes rituels. Le portail peut dès lors être considéré comme une sorte de re-jeu, dans lequel les Amiénois peuvent se reconnaître et se trouver ainsi, par la vertu de l'image, intégrés au grandiose programme iconographique de la cathédrale ${ }^{1}$. Le portail saint Honoré provoque une réflexion d'un autre ordre, centré sur l'efficacité respective des images et des ornamenta. La représentation d'une femme recouvrant la vue en appliquant un drap d'autel sur ses yeux ne fait pas qu'authentifier les capacités miraculeuses des objets liturgiques contenus dans la cathédrale: dans le même temps, c'est la puissance salvatrice de l'ecclesia, avec l'ensemble des images qu'elle supporte, qui est renforcée par la présence de tels objets dans son sein. Image et liturgie forment dans ce cas un couple dont l'association renforce les capacités propres à chacun des deux termes.

Pour finir, cet ouvrage s'impose comme un objet de choix, particulièrement utile par la qualité des images et des plans qu'il propose (même si, pour le prix, on serait en droit d'exiger une meilleure qualité, qui laisse parfois à désirer, par exemple p. 56-57 et 166-167) ; il s'agit de fait d'un travail d'importance, qui prend acte d'un certain nombre de changements intervenus dans l'histoire de l'art et des images au cours de ces dernières années. L'ensemble des contributions oblige à considérer la création des formes (et plus encore leur réception) au sein de dispositifs complexes où interviennent et interagissent architecture, peinture murale, sculpture, art mobilier et témoignages écrits de rituels. L'ampleur de la proposition est renforcée par le choix d'une véritable perspective européenne (autant dans le choix des auteurs que du corpus), qui soulage $\mathrm{du}$ caractère étriqué des histoires de l'art nationales et patrimoniales. La voie ouverte par cet ouvrage est loin d'être entièrement parcourue, un usage plus systématique de la littérature liturgique et surtout des objets mobiliers, qu'ils soient porteurs d'images ou non, apporteront sans doute dans les années à venir une compréhension profondément renouvelée de ces dispositifs.

\section{NOTES}

1. Sur cette notion de re-jeu, voir B. D'HAINAUT-ZVENY, « Des compétences changeantes. Petit essai sur l'évolution des rôles assignés aux images dans les retables romans, gothiques et renaissants ", dans G. BARTholeyns et T. GOLSENNE éd., La Performance des images, Bruxelles, 2009, p. 87-99. 


\section{AUTEURS}

\section{PIERRE-OLIVIER DITTMAR}

GAHOM, EHESS 